\title{
Bedside POCUS during ward emergencies is associated with improved diagnosis and outcome: an observational, prospective, controlled study
}

\author{
Laurent Zieleskiewicz ${ }^{1,6^{*}+}$ (D) Alexandre Lopez ${ }^{1 \dagger}$, Sami Hraiech² ${ }^{2}$ Karine Baumstarck ${ }^{3}$, Bruno Pastene ${ }^{1}$, \\ Mathieu Di Bisceglie ${ }^{4}$, Benjamin Coiffard ${ }^{2}$, Gary Duclos ${ }^{1}$, Alain Boussuges ${ }^{5,6}$, Xavier Bobbia ${ }^{7}$, Sharon Einav ${ }^{8}$, \\ Laurent Papazian² and Marc Leone
}

\begin{abstract}
Background: Rapid response teams are intended to improve early diagnosis and intervention in ward patients who develop acute respiratory or circulatory failure. A management protocol including the use of a handheld ultrasound device for immediate point-of-care ultrasound (POCUS) examination at the bedside may improve team performance. The main objective of the study was to assess the impact of implementing such a POCUS-guided management on the proportion of adequate immediate diagnoses in two groups. Secondary endpoints included time to treatment and patient outcomes.

Methods: A prospective, observational, controlled study was conducted in a single university hospital. Two teams alternated every other day for managing in-hospital ward patients developing acute respiratory and/or circulatory failures. Only one of the team used an ultrasound device (POCUS group).

Results: We included 165 patients (POCUS group 83, control group 82). Proportion of adequate immediate diagnoses was $94 \%$ in the POCUS group and $80 \%$ in the control group $(p=0.009)$. Time to first treatment/intervention was shorter in the POCUS group (15 [10-25] min vs. 34 [15-40] min, $p<0.001)$. In-hospital mortality rates were $17 \%$ in the POCUS group and $35 \%$ in the control group ( $p=0.007$ ), but this difference was not confirmed in the propensity score sample (29\% vs. $34 \%, p=0.53)$.

Conclusion: Our study suggests that protocolized use of a handheld POCUS device at the bedside in the ward may improve the proportion of adequate diagnosis, the time to initial treatment and perhaps also survival of ward patients developing acute respiratory or circulatory failure.

Clinical Trial Registration NCT02967809. Registered 18 November 2016, https://clinicaltrials.gov/ct2/show/NCT0296780 9.
\end{abstract}

*Correspondence: Laurent.zielekiewicz@ap-hm.fr

${ }^{\dagger}$ Laurent Zieleskiewicz and Alexandre Lopez have contributed equally to

this work

${ }^{\dagger}$ Laurent Zieleskiewicz and Alexandre Lopez are joint first authors

${ }^{1}$ Assistance Publique Hôpitaux de Marseille, Department

of Anesthesiology and Intensive Care, Hôpital Nord, Aix Marseille

University, 13015 Marseille, France

Full list of author information is available at the end of the article

(c) The Author(s) 2021. This article is licensed under a Creative Commons Attribution 4.0 International License, which permits use, sharing, adaptation, distribution and reproduction in any medium or format, as long as you give appropriate credit to the original author(s) and the source, provide a link to the Creative Commons licence, and indicate if changes were made The images or other third party material in this article are included in the article's Creative Commons licence, unless indicated otherwise in a credit line to the material. If material is not included in the article's Creative Commons licence and your intended use is not permitted by statutory regulation or exceeds the permitted use, you will need to obtain permission directly from the copyright holder. To view a copy of this licence, visit http://creativecommons.org/licenses/by/4.0/. The Creative Commons Public Domain Dedication waiver (http://creativecommons.org/publicdomain/zero/1.0/) applies to the data made available in this article, unless otherwise stated in a credit line to the data. 
Keywords: POCUS, In-hospital emergencies, Rapid response team, Handheld ultrasound device

\section{Background}

Patients admitted to conventional hospital wards often develop acute respiratory and/or circulatory failure which requires treatment by a rapid response team (RRT) [1]. Early diagnosis and intervention may prevent further deterioration, resulting in reduced in-hospital mortality [2,3], yet the outcomes of patients treated by RRT remain highly variable [4]. Previous studies have shown that during emergencies, point-of-care ultrasound (POCUS) facilitates the identification of causes of respiratory and circulatory failure $[5,6]$. In observational studies, POCUS seems to improve the likelihood of early diagnosis in such events and decrease the time to administration of treatment [7], including in patients with both acute respiratory and acute circulatory failure $[8,9]$.

"Handheld ultrasound devices" (HHUD) are the new generation of ultrasound devices. Their diagnostic capabilities have been validated against conventional ultrasound devices for focused echocardiography, lung ultrasound and deep venous thrombosis detection [1012]. Such devices have the advantage of being easy to use by RRT at the bedside [13], but the clinical value of such use remains unclear.

We hypothesized that introduction of a RRT treatment protocol based on bedside cardiac and pulmonary POCUS findings in the ward would improve diagnosis, time to treatment and outcomes for patients developing acute respiratory and/or circulatory failures in the ward. The primary aim of this study was to assess the impact of implementing such a POCUS-guided management on the proportion of adequate immediate diagnoses (i.e., during the event) in relation to the definitive diagnosis (i.e., upon discharge). The secondary aims were to assess the impact of POCUS on the time to immediate diagnosis, the appropriateness of care, the need for additional diagnostic tests and patient outcomes.

\section{Methods \\ Design}

A single-center, prospective, observational, controlled study was performed at the North Hospital of Marseille (Assistance Publique-Hôpitaux Marseille) from November 2016 to November 2018. In accordance with French law [14], patients were informed regarding the use of their data for publication. Because all strategies used in this study were considered standard of care, the study was observational and informed consent was not required. The study was compliance with the Strengthening the Reporting of Observational Studies in Epidemiology (STROBE) recommendations [15].

\section{Characteristics of participants}

During the study period, all consenting adults hospitalized in medical or surgical wards and developing respiratory and/or circulatory failure justifying placement of a call to the RRT were prospectively included. Exclusion criteria were age below 18 years, pregnancy, cardiac arrest, technical limitations to the performance of ultrasound examination in the ward (e.g., surgical dressings, anatomical abnormalities), lung or cardiac transplant, RRT calling for a neurological failure, RRT calling by the emergency department and impossible follow-up.

In-hospital calls to the RRT were attended by the RRT of two intensive care units (ICUs) in the hospital which alternated every other day. In our institution, the RRT is comprised of a senior ICU physician, an ICU resident and a medical student. The two ICUs cover RRT activity in turns regardless of the study; therefore, a decision was undertaken that one would serve as the POCUS group and the other would serve as the control group (no POCUS using by the control RRT). A protocol for guiding patient treatment based on POCUS findings was developed and made available only to the RRT assigned to the POCUS group (Fig. 1a, b).

\footnotetext{
(See figure on next page.)

Fig. 1 POCUS-guided managements: ultrasound protocol. (a) Circulatory failure protocol. Use of the HHUD (Vscan Dual Probe extend ${ }^{\mathrm{TM}}$, General Electric Healthcare): Cardiac assessment with sector transducer to access the LV and RV function: (1) subcostal view or (2) apical four-chamber view-Pulmonary assessment with the linear transducer: (1) anterior chest based between the clavicle to the diaphragm and the mid-axillary line (2) pleural bases. Abbreviations: EFLV, ejection fraction left ventricular; IVC, inferior vena cave; LV, left ventricular; RA, right auricle; RV, right function. (b) Respiratory failure protocol. Use of the HHUD (Vscan Dual Probe extend ${ }^{\mathrm{TM}}$, General Electric Healthcare): Pulmonary assessment with the linear transducer: (1) anterior chest based between the clavicule to the diaphragm and the mid-axillary line (2) pleural bases. Cardiac assessment with the sector transducer to assess the right ventricular function: (1) subcostal view or $n$ apical four-chamber view. Deep venous assessment with the linear transducer to identify thrombosis: femoral and popliteal veins. The visual EFLV helps to differentiate between cardiogenic edema (EFLV altered) and acute interstitial lung disease (non-cardiogenic edema) (EFLV not altered). Abbreviations: EFLV, ejection fraction left ventricular; COPD, chronic obstructive pulmonary disease
} 


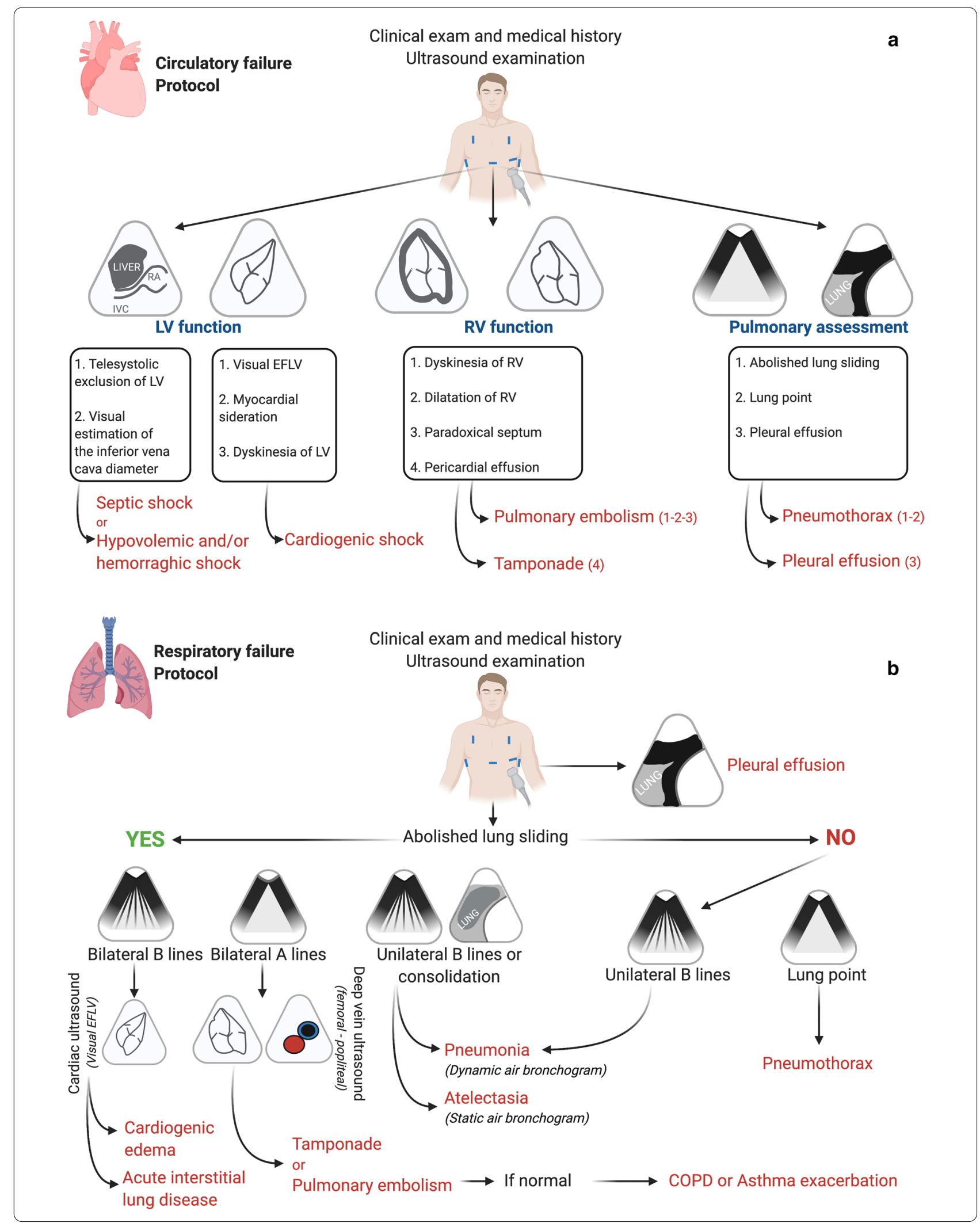




\section{Training and education of physicians}

During the three-hour training session (by a level 3 senior physician [16]), typical ultrasound signs of the main causes of acute respiratory and circulatory failure were depicted. This was followed by classroom presentation of practical interactive case reports and a hands-on scenario-based workshop, during which the attendees were required to follow the treatment protocol with a portative ultrasound device. In our department, ultrasound training sessions are offered to both senior and junior physicians twice a year by a level 3 certified senior physician. All senior physicians have at least a level 2 in thoracic ultrasound.

\section{Study protocol}

Calls to the RRT are placed by the physicians in charge of the patients on the ward. Such calls are typically made after an initial assessment by the senior treating physician on location for the presence of organ failures. The reason for RRT intervention (acute respiratory or circulatory failure), the indication for hospital admission and patient features are recorded routinely. Definitions of all terms used within the manuscript are summarized in Additional file 1: Table 1.

\section{Clinical assessment}

Each patient underwent a standard medical examination by the RRT. This included taking the medical history, performance of a circulatory, respiratory and neurological assessment, monitoring of vital signs, blood testing and conduction of any additional tests judged necessary by the physician in charge. Control patients received treatment according to team assessment based on these data. Study patients also underwent a structured POCUS examination to direct care.

\section{POCUS examination in the ward}

Patients in the POCUS group underwent an immediate bedside POCUS examination in the ward using a handheld ultrasound device (Vscan Dual Probe Extend ${ }^{\mathrm{TM}}$, GE Healthcare). The ultrasound examination included focused cardiac and pulmonary echography and imaging of the deep veins when deemed necessary. The echogenicity of each patient was graded as poor, moderate or good. The examination was interpreted by an ICU senior physician. The POCUS protocol is detailed in Fig. 1a, b [17-20].

\section{Immediate diagnosis: management in the ward}

Following clinical assessment at the bedside, each RRT selected a diagnosis from a pre-prepared list (the immediate diagnosis). This list was established by the investigators prior to study initiation and validated by the senior physicians involved in the study [7, 9, 21] (Additional file 2: Table 2). In both groups, the time to immediate diagnosis and the time to first treatment were recorded. The number of interventions performed and the number of supplementary tests ordered per patient were collected during review of the medical files for both groups. Each RRT also recorded the treatment the patient received immediately on location and supplemental workup if such was prescribed in the ward (e.g., imaging modalities) as well as patient placement following triage by the RRT (ward, immediate surgery, emergency room or ICU). Patients requiring critical care were admitted to one of the two ICUs according to bed availability.

\section{Definitive diagnosis}

The definitive cause of respiratory and/or circulatory failure was determined following retrospective review of all patient medical files. Two physicians from each team, blinded of the patient group and the initial diagnoses made at the bedside (BP and $\mathrm{SH}$ ) independently reviewed all the documentation. This included nursing and medical follow-ups, physical examinations and blood and imaging tests. Using this documentation, each determined the definitive cause of deterioration (i.e., the "definitive diagnosis"). In case of disagreement between the two reviewers or if doubt arose, a third expert was consulted (ML) and a consensus was looked for. In several cases, more than one cause was deemed to have led to patient deterioration. In such cases, all of the causes were listed by the reviewing physicians. For determining the percent of adequate diagnosis between the initial diagnosis made at the bedside and the definitive diagnosis, the list of definitive diagnoses reached by the reviewing physicians was compared to the diagnoses documented on the research forms on location of the event. If even one of the diagnoses on the two lists correlated, the diagnosis made at the bedside was classified as correct (Additional file 3: Table 3).

\section{Sample size considerations}

Overall 160 patients were required (80 per group) to detect an absolute difference of $15 \%$ between the groups in the proportion of adequate immediate diagnoses in relation to the definitive diagnoses, with an alpha risk and power of $5 \%$ and $80 \%$, respectively. This included an assumed $10 \%$ dropout.

\section{Statistical analysis}

Baseline patient and treatment characteristics were compared between the control group and the POCUS group. The percent agreement between the immediate diagnoses made at the bedside and the definitive diagnoses (primary outcome) was compared between groups using the 
$\chi 2$ test. Comparisons of secondary outcomes were performed using the $\chi 2$ or Fischer's exact tests for qualitative variables, and the Student T or Mann-Whitney tests for quantitative variables based on variable distribution.

As sensitivity analysis, multivariate analysis was performed using logistic regressions (enter method) to determine the adjusted effect of the treatment group on in-hospital mortality. Variables were selected for model inclusion if they were of clinical interest and/or if they had a threshold $p$ value $<0.1$ in univariate analysis. The final models are presented with the odds ratios (ORs) and 95\% confidence intervals (95\% CIs). A predefined subgroup analysis was performed according to the indication for calling the RRT (respiratory vs. circulatory). Statistical analyses were performed using SPSS (IBM SPSS Statistics version 20 for windows).

To reduce selection bias, we performed a propensity score matching. Eight covariates were included in the propensity score model (Simplified Acute Physiology Score (SAPS) II, mottling's presence, respiratory rate, pulse oximetry, need for oxygen therapy, chronic heart failure, chronic obstructive pulmonary disease, and sex). The matching was performed using the R MatchIt package and was based on the nearest neighbor with caliper matching (compiled with version 3.5.3; Feather Spray Copyright (C) 2018 The R Foundation for Statistical Computing). Accuracy of the score was assessed using histograms of propensity scores before and after matching and standardized mean differences (SMD) of the covariates. The matching identified 42 POCUS and 73 control individuals. All the details of the propensity scores are provided in Additional file 4: Additional Method.

No data imputation was performed. All the tests were two-sided. Statistical significance was defined as $p<0.05$.

\section{Results}

During the study period, 486 patients were screened. Among them, 165 patients were enrolled: 83 to the POCUS group and 82 to the control group (Fig. 2). The patients enrolled in the two groups differed only in the rate of mottling (10\% in the POCUS group versus $27 \%$ in the control group, respectively, $p=0.02)$, the median oxygen flow rates (6, IQR [2-15] versus 12 , IQR [5-15] $1 /$ min, respectively, $p=0.003)$ and mean arterial pressure (MAP) (93, IQR [72-113] versus 82, IQR [70-100] $\mathrm{mmHg}$, respectively, $p=0.02$ ). The indications for calling the RRT were similar in the two groups (Table 1). In both groups, several cases retained more than one final diagnosis even after three reviewing rounds. The definitive diagnoses retained were similar in the two groups. Senior and junior physicians of the two teams shared the same level of expertise (Additional file 5: Table 4).

\section{Primary outcome}

The proportion of immediate adequate diagnosis at the bedside in the ward was $94 \%$ in the POCUS group and $80 \%$ in the control group $(p=0.009)$ (Table 2$)$. In the matched cohort, the proportion of immediate adequate diagnosis was $93 \%$ in the POCUS group and $78 \%$ in the control group $(p=0.04)$ (Additional file 5: Table 5A).

\section{Secondary outcomes}

POCUS echogenicity was rated poor for cardiac assessment in $9 \%$ of cases and for lung assessment in $11 \%$ of cases.

The time to immediate diagnosis at the bedside was similar in the two groups. However, the median time to first treatment/intervention was $15 \mathrm{~min}$ [IQR 10-25] in the POCUS group and 34 min [IQR 15-40] in the control group $(p<0.001)$. This finding was most pronounced in the subgroup of patients with acute respiratory failure $(p<0.001)$. The proportion of appropriate intervention at the bedside was similar in the two groups. The POCUS group required less supplemental workup than the control group (one additional test [IQR 1-2] versus two additional tests [IQR 1-3], $p<0.001$ ) (Table 2).

The rate of patient triage to ICU admission was 55\% in the POCUS group and $72 \%$ in the control group $(p=0.15)$ (Table 3). For those patients admitted to the ICU, the median length of ICU stay was 3 days [IQR 2-7] in the POCUS group and 5 days [IQR 3-10] days in the control group $(p=0.01)$. The median hospital length of stay did not differ in the POCUS group (16 days [IQR 9-25] and in the control group (16 days [IQR 9-28] days, $p=0.44)$ ) (Table 2). All the details of the propensity scores are provided in Additional file 5: Table 5A.

The median number of interventions performed per case during bedside management in ward was three [IQR $2-4]$ in the POCUS group and four [IQR 3-4] in the control group $(p<0.001)$. Patients in the POCUS group also received less invasive mechanical ventilation $(p=0.02)$, systemic steroids $(p=0.02)$ and vasopressors $(p<0.001)$ than patients in the control group (Table 3). All the details of the propensity scores are provided in Additional file 5: Table 5B.

ICU mortality rates were $11 \%$ in the POCUS group and $25 \%$ in the control group $(p=0.04)$. In-hospital mortality rates were $17 \%$ in the POCUS group and $35 \%$ in the control group $(p=0.007)$ (Table 4$)$. These differences were not found in the propensity cohort $(p=0.79$ and $p=0.53$, respectively) (Additional file 5: Table 5C). The POCUS effect on in-hospital mortality was assessed after adjustment for the main confounding factors: The effect remains significant for most models (sensitivity analyses, Additional file 7: Table 6). 


293 Excluded because they did not meet inclusion
criteria
45 Dead
73 Transfert in other hospital
36 Mistake of calling
82 Calling by Emergency Physician
21 Cardiac arrest
36 Coma

28 Excluded 12 Missing data 2 Transplants

5 Twice inclusion

7 Immediate withholding and withdrawal of life-

2 cardiac arrest with no final diagnosis
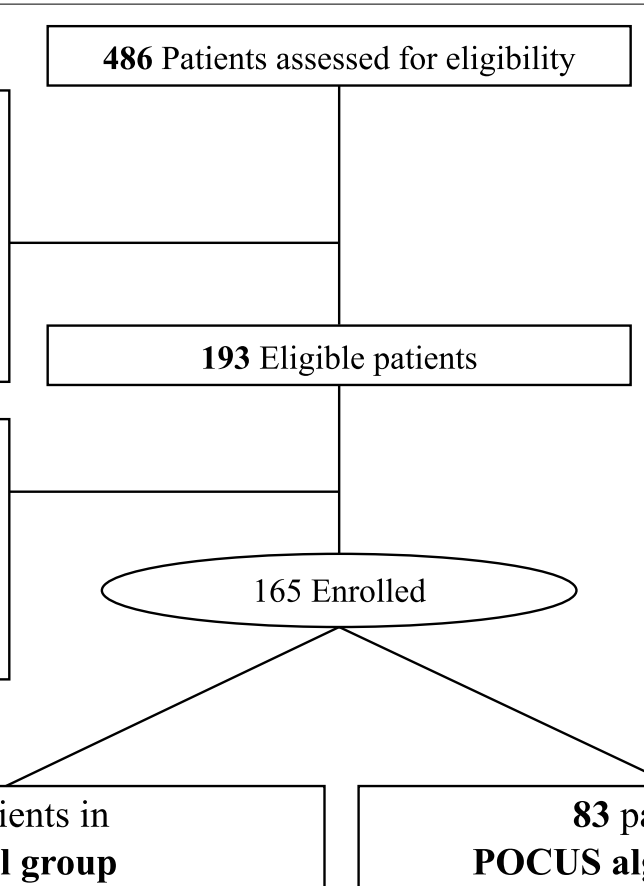

82 patients in control group

82 Primary and secondary outcomes collected

Immediate adequate diagnosis: 66 Patients $(80 \%)^{*}$

Secondary outcome:

In-Hospital mortality : 29 patients $(35 \%)^{* *}$

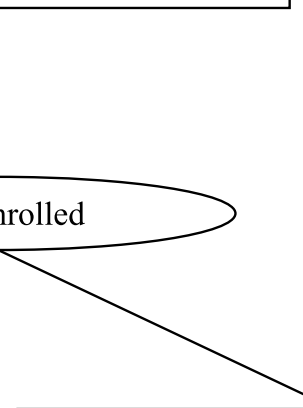

sustaining treatments
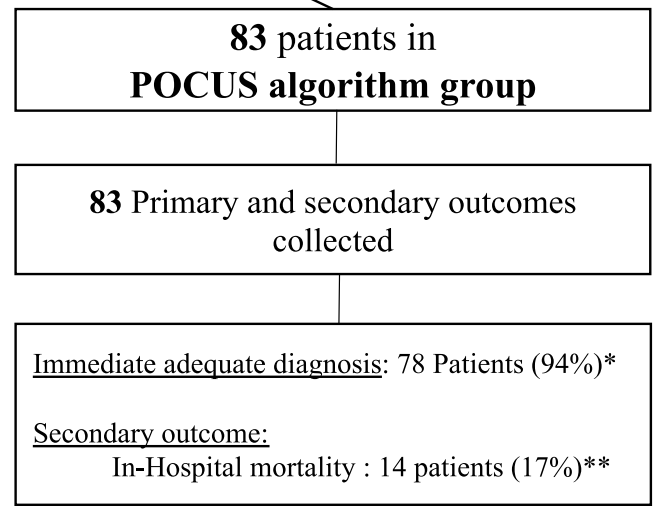

Fig. 2 Flowchart. ${ }^{*} p=0.009,{ }^{* *} p=0.007$. Details of excluded patients by groups: control group: 4 immediate withholding and withdrawal of life-sustaining treatments; 2 twice inclusion; 4 missing data; 2 transplant's patients; 1 cardiac arrest. POCUS group: 3 immediate withholding and withdrawal of life-sustaining treatments; 8 missing data; 3 twice inclusion; 1 cardiac arrest

\section{Discussion}

In this single-center prospective, controlled trial, patients for whom the RRT was called underwent ward treatment that was allocated to be either POCUSguided management directed or physician judgment directed. Patients in the POCUS group had a higher proportion of adequate immediate diagnosis, were treated more rapidly and had higher survival rates than patients treated conventionally.

A seminal study showed the potential diagnostic impact of lung ultrasound in a cohort of 260 patients admitted to the ICU for acute respiratory failure [18]. Since then, POCUS has repeatedly been proven superior to the combination of physical examination and chest X-ray for diagnosis of patients with acute respiratory failure $[5,9,23,24]$. In patients with acute circulatory failure, POCUS has a high diagnostic accuracy $[6,25]$ and can change therapeutic management [26-28]. Despite the major differences between the ICU and the emergency department in terms of staff training and resources, similar results are consistently found in both with regards to POCUS [9, 23, 25]. Our study focused on patients hospitalized in conventional wards where resources are often limited, thereby demonstrating the advantage of POCUS directed treatment in an entirely different environment.

POCUS can be performed by the clinician at the bedside using a handheld device. Compared to other potentially relevant tests, it does not require that a device be brought by another person to the bedside, it is independent of external services and it is noninvasive. POCUS has been described as the fifth pillar of physical examination [29] because it provides more physiological information 
Table 1 Baseline participants characteristics ${ }^{a}$

\begin{tabular}{|c|c|c|c|}
\hline Variables & Control group $n=82$ & POCUS group $n=83$ & $P$ value \\
\hline Men & $48(59)$ & $55(66)$ & 0.31 \\
\hline Age, median [IQR] years & $67[57-77]$ & 70 [59-81] & 0.49 \\
\hline \multicolumn{4}{|c|}{ Indication for placing the call to the RRTS } \\
\hline Respiratory & $56(68)$ & $61(74)$ & \multirow[t]{2}{*}{0.46} \\
\hline Circulatory & $26(32)$ & $22(26)$ & \\
\hline \multicolumn{4}{|c|}{ Indication for hospital admission } \\
\hline Cardiac & $10(12)$ & $3(4)$ & \multirow[t]{11}{*}{0.27} \\
\hline Respiratory & $30(37)$ & $23(28)$ & \\
\hline Neurologic & $2(2)$ & $7(9)$ & \\
\hline Digestive & $8(10)$ & $8(10)$ & \\
\hline Obstetrical & $1(1,5)$ & 0 & \\
\hline Infectious & $2(2,5)$ & $2(2)$ & \\
\hline General & $4(5)$ & $4(5)$ & \\
\hline Surgery & $24(29)$ & $28(34)$ & \\
\hline Urologic & 0 & $2(2)$ & \\
\hline Vascular & $1(1)$ & $3(4)$ & \\
\hline Traumatic & 0 & $3(4)$ & \\
\hline \multicolumn{4}{|l|}{$B M I(>25), \mathrm{kg} / \mathrm{m}^{2}$} \\
\hline & $41(50)$ & $39(47)$ & 0.70 \\
\hline \multicolumn{4}{|l|}{ ASA score } \\
\hline$|-| \mid$ & $23(28)$ & $23(28)$ & \multirow[t]{3}{*}{0.99} \\
\hline III & $47(57)$ & $48(58)$ & \\
\hline IV-V & $12(15)$ & $12(14)$ & \\
\hline \multicolumn{4}{|l|}{ SAPS $\|^{b}$, median $[I Q R]$} \\
\hline & 46 [34-57] & $40[33-52]$ & 0.51 \\
\hline \multicolumn{4}{|l|}{ Comorbidities } \\
\hline Allergy & $9(11)$ & $8(10)$ & 0.78 \\
\hline Coronary disease & $22(27)$ & $23(28)$ & 0.89 \\
\hline Heart failure & $19(23)$ & $27(32)$ & 0.18 \\
\hline Chronic kidney disease & $11(13)$ & $19(22)$ & 0.16 \\
\hline Cancer & $28(34)$ & $26(31)$ & 0.70 \\
\hline Immunodepression & $10(12)$ & $13(15)$ & 0.52 \\
\hline Dementia & $5(6)$ & $3(4)$ & 0.49 \\
\hline Stroke & $8(9,8)$ & $9(11)$ & 0.82 \\
\hline Epilepsy & $5(6)$ & $1(1)$ & 0.12 \\
\hline Diabetes & $13(16)$ & $21(25)$ & 0.13 \\
\hline Liver disease & $6(7)$ & $6(7)$ & 0.98 \\
\hline COPD & $36(44)$ & $30(36)$ & 0.31 \\
\hline Habitual smoker & $42(51)$ & $34(41)$ & 0.19 \\
\hline \multicolumn{4}{|l|}{ Definitive diagnosis ${ }^{c}$} \\
\hline Exacerbation of asthma & $3(5)$ & $3(5)$ & 0.60 \\
\hline Septic shock & $14(17)$ & & $14(17)$ \\
\hline Pneumonia & $18(22)$ & $7(8)$ & \\
\hline Cardiogenic edema & $10(12)$ & $13(16)$ & \\
\hline Hemorrhagic shock & $7(8)$ & $4(5)$ & \\
\hline Atelectasis & $1(1)$ & $4(5)$ & \\
\hline Exacerbation of COPD & $5(6)$ & $6(7)$ & \\
\hline Pneumothorax & $1(1)$ & $1(1)$ & \\
\hline Pleural effusion & $3(5)$ & $4(5)$ & \\
\hline
\end{tabular}


Table 1 (continued)

\begin{tabular}{|c|c|c|c|}
\hline Variables & Control group $n=82$ & POCUS group $n=83$ & $P$ value \\
\hline Acute interstitial lung disease (non-cardiogenic edema) & $4(5)$ & $7(8)$ & \\
\hline Pulmonary embolism & $2(2)$ & $2(2)$ & \\
\hline Cardiogenic shock & $2(2)$ & $2(2)$ & \\
\hline Hypovolemic shock & $1(1)$ & $1(1)$ & \\
\hline Tamponade & $1(1)$ & $2(2)$ & \\
\hline Exacerbation of COPD/cardiogenic edema & 0 & $1(1)$ & \\
\hline Pneumonia/cardiogenic edema & 0 & $1(1)$ & \\
\hline Pneumonia/acute interstitial lung disease (non-cardiogenic edema) & $1(1)$ & $3(5)$ & \\
\hline Exacerbation of COPD/pneumothorax & $2(2)$ & 0 & \\
\hline Normal & 0 & $2(2)$ & \\
\hline Othert & $7(9)$ & $6(8)$ & \\
\hline \multicolumn{4}{|l|}{ Urine volume $24 \mathrm{~h}$ before inclusion (ml/day) } \\
\hline$<200$ & $6(7)$ & $4(5)$ & 0.58 \\
\hline$<500$ & $29(35)$ & $24(29)$ & \\
\hline$>500$ & $37(45)$ & $40(48)$ & \\
\hline$>1000$ & $10(12)$ & $15(18)$ & \\
\hline Mottling $^{d}$ & $22(27)$ & $10(12)$ & 0.02 \\
\hline Respiratory distress syndrome & $49(60)$ & $54(65)$ & 0.48 \\
\hline Abnormal chest auscultation ${ }^{e}$ & $62(76)$ & $59(71)$ & 0.51 \\
\hline Thorax X-ray $<24 \mathrm{~h}$ & $35(42)$ & $38(46)$ & 0.69 \\
\hline MAP, median [IQR], mmHg & $82[70-100]$ & $93[72-113]$ & 0.02 \\
\hline Heart rate, median [IQR], bpm* & $100[80-120]$ & $107[85-130]$ & 0.46 \\
\hline Serum lactate median [IQR], mmol/I & $2[1-3]$ & $2[1-3]$ & 0.95 \\
\hline Hemoglobin, median [IQR], g/dl & $11[9-13]$ & $11[9-12]$ & 0.18 \\
\hline Serum creatinine, median $[\mid \mathrm{QR}], \mathrm{mmol} / \mathrm{L}$ & $79[52-125]$ & $82[62-132]$ & 0.51 \\
\hline Total bilirubin, median [IQR] umol/I & $10[8-16]$ & $10[8-16]$ & 0.91 \\
\hline Serum potassium, median [IQR], mmol/L & $4[4,5]$ & $4[4,5]$ & 0.41 \\
\hline Volemic expansion at the bedside, median [IQR], $\mathrm{ml} / \mathrm{kg}$ & $3[0-10]$ & $4[0-12]$ & 0.73 \\
\hline Respiratory rate, median [IQR] bpm** & $27[20-35]$ & $25[20-35]$ & 0.51 \\
\hline SpO2, median [IQR], \% & $92[89-96]$ & 94 [90-97] & 0.16 \\
\hline $\mathrm{PaO} 2 / \mathrm{FiO} 2$ ratio, median [IQR] & $186[121-284]$ & $203[136-355]$ & 0.28 \\
\hline Glasgow score, median [IQR] & $15[2-15]$ & $15[14,15]$ & 0.08 \\
\hline BNP, median [IQR] pg/ml & 306 [129-584] & 188 [62-833] & 0.61 \\
\hline Oxygen rate flow, median [IQR], L/min & $12[5-15]$ & $6[2-15]$ & 0.003 \\
\hline \multicolumn{4}{|l|}{ Orientation after inclusion } \\
\hline Immediate ICU & $59(72)$ & $49(55)$ & 0.15 \\
\hline Operative room & $5(6)$ & $7(8)$ & \\
\hline Emergency room & $4(5)$ & $9(12)$ & \\
\hline Ward & $14(17)$ & $21(25)$ & \\
\hline
\end{tabular}

BMI, body mass index; ASA, Score of American Society of Anesthesiologists; SAPS II, Simplified Acute Physiology Score II; COPD, chronic obstructive pulmonary disease; MAP, median arterial pressure; $\mathrm{SpO}$, pulse oximetry; $\mathrm{PaO} 2 / \mathrm{FiO} 2$ ratio, ratio of arterial oxygen partial to fractional inspired oxygen; $\mathrm{BNP}$, brain natriuretic peptide; bpm*: beats per minute; $b p p m^{* *}$ : breaths per minute; IQR, interquartile range; RRT, rapid response team

a Data are expressed as No (\%) of participants unless otherwise indicated. At the bedside

b The SAPS II ranges from 0 to 163, with higher scores indicating higher risk of mortality. A patient with a score of 40 has an estimated mortality risk of $30 \%$

c Diagnosis established after medical files and supplementary workup

d Only presence or absence of mottlings

e Abnormal was defined like pathological auscultation

† Panic attack (1), anaphylaxia (2), bronchospasm (1), hemoptysis (1), intraalveolar hemorrhage (1), laryngeal dyspnea (1), morphine surdosage (1), pulmonary hypertension crisis (1), tracheal decannulation (1), neurological failure (1) and valve thrombosis (1) 
Table 2 Primary and secondary outcomes of patients ${ }^{a}$

\begin{tabular}{|c|c|c|c|}
\hline Outcome category & Control group $n=82$ & POCUS group $n=83$ & $P$ value \\
\hline \multicolumn{4}{|l|}{ Primary outcome } \\
\hline \multicolumn{4}{|c|}{ Immediate adequate diagnosis } \\
\hline General & $66(80)$ & $78(94)$ & 0.009 \\
\hline Circulatory & $24 / 26(92)$ & $21 / 22(95)$ & 0.65 \\
\hline Respiratory & $42 / 56(75)$ & $57 / 61(93)$ & 0.006 \\
\hline \multicolumn{4}{|l|}{ Secondary outcomes } \\
\hline \multicolumn{4}{|c|}{ Appropriate intervention in ward } \\
\hline General & $72(88)$ & $77(93)$ & 0.28 \\
\hline Circulatory & $22 / 26(84)$ & 20/21 (95) & 0.36 \\
\hline Respiratory & $48 / 56(86)$ & $57 / 62(92)$ & 0.28 \\
\hline \multicolumn{4}{|c|}{ Time to immediate diagnosis, median [IQR], min } \\
\hline General & $11[5-20]$ & $10[5-15]$ & 0.16 \\
\hline Circulatory & $14[7-20]$ & $10[6-20]$ & 0.94 \\
\hline Respiratory & $16[5-20]$ & $10[3-30]$ & 0.13 \\
\hline \multicolumn{4}{|c|}{ Time to first treatment/intervention, median [IQR], min } \\
\hline General & $34[15-40]$ & 15 [10-25] & 0.00003 \\
\hline Circulatory & $22[14-45]$ & $15[11-28]$ & 0.26 \\
\hline Respiratory & $30[15-38]$ & $15[20-61]$ & 0.00003 \\
\hline \multicolumn{4}{|c|}{ Number of interventions, median [IQR], min } \\
\hline General & $4[3,4]$ & $3[2-4]$ & 0.0009 \\
\hline Circulatory & $4[3,4]$ & $3[2-4]$ & 0.07 \\
\hline Respiratory & $4[3,4]$ & $3[2-4]$ & 0.005 \\
\hline \multicolumn{4}{|c|}{$\begin{array}{l}\text { Number of supplementary examinations during first day, median } \\
\text { [IQR] }\end{array}$} \\
\hline General & $2[1-3]$ & $1[1.2]$ & 0.00002 \\
\hline Circulatory & $2[1-3]$ & $2[1,2]$ & 0.009 \\
\hline Respiratory & $2[2,3]$ & $1[1,2]$ & 0.0006 \\
\hline \multicolumn{4}{|c|}{ ICU length of stay, median [IQR], days } \\
\hline General & $5[3-10]$ & $3[2-7]$ & 0.01 \\
\hline Circulatory & $4[2-6]$ & $2[1-7]$ & 0.23 \\
\hline Respiratory & $5[3-13]$ & $3[2-7]$ & 0.01 \\
\hline \multicolumn{4}{|c|}{ Hospital length of stay, median [IQR], days } \\
\hline General & $16[9-25]$ & $16[9-28]$ & 0.44 \\
\hline Circulatory & $13[8-31]$ & $10[6-29]$ & 0.35 \\
\hline Respiratory & 18 [9-29] & $17[10-28]$ & 0.72 \\
\hline
\end{tabular}

IQR: interquartile range

a Data are expressed as no. (\%) of participants unless otherwise indicated

than do clinical examination, blood gases and electrocardiography combined [30].

Improved diagnostic performance is an explanation for the earlier treatment and reduced number of supplementary examinations observed in the POCUS group when compared with the control group. During emergencies, targeted POCUS screening can include assessment of cardiac function, filling and tamponade, gross valvular function, venous filling, pleural sliding and fluid accumulation as well as deep venous return [10, 31-33]. If a diagnosis is made using POCUS, on-site intervention can be targeted to treat the abnormal finding, thus decreasing the number of supplementary examinations required and also reducing the likelihood of redundant interventions. We did not measure the cost of treatment. However, it follows that a reduction in resource use would also reduce costs.

Our study suggests an association between ICU and in-hospital mortalities and structured use of POCUS during ward emergencies although these findings were not confirmed after matching. To the best of our knowledge, no RCT has shown such an association, and 
Table 3 Administrated treatments by the rapid response teams at the bedside in POCUS group versus the physician judgment (control) treatment groups ${ }^{a}$

\begin{tabular}{llll}
\hline & $\begin{array}{l}\text { Control group } \\
\boldsymbol{n = 8 2}\end{array}$ & $\begin{array}{l}\text { POCUS group } \\
\boldsymbol{n = 8 3}\end{array}$ & $\begin{array}{l}\boldsymbol{P} \text { value } \\
\text { Drainage }\end{array}$ \\
Volemic expansion & $8(10)$ & $14(17)$ & 0.18 \\
Diuretics & $40(49)$ & $29(35)$ & 0.07 \\
Nitrates & $13(16)$ & $16(19)$ & 0.56 \\
Noninvasive ventilation & $2(2)$ & $7(8)$ & 0.16 \\
Oxygen therapy & $15(18)$ & $21(25)$ & 0.27 \\
Invasive ventilation & $58(71)$ & $53(64)$ & 0.34 \\
Aerosol bronchodilators & $20(24)$ & $9(11)$ & 0.02 \\
Aerosol corticoids & $22(27)$ & $21(25)$ & 0.82 \\
Systemic corticoid & $9(11)$ & $4(5)$ & 0.14 \\
Antibiotic & $13(16)$ & $4(5)$ & 0.02 \\
Curative anticoagulation & $43(52)$ & $28(34)$ & 0.02 \\
Aspirin & $4(5)$ & $5(6)$ & 1 \\
Inotrope & $2(2)$ & 0 & 0.25 \\
Vasopressor & $3(4)$ & $3(4)$ & 1 \\
Antiarrhythmic & $29(35)$ & $7(8)$ & 0.00002 \\
Transfusion & $1(1)$ & $1(1)$ & 1 \\
Physiotherapy & $7(9)$ & $3(4)$ & 0.21 \\
\hline
\end{tabular}

${ }^{a}$ Data are expressed as no. (\%) of participants

Table 4 In-hospital and ICU mortality rates in POCUS group versus control group ${ }^{a}$

\begin{tabular}{|c|c|c|c|}
\hline & $\begin{array}{l}\text { Control group } \\
n=82\end{array}$ & $\begin{array}{l}\text { POCUS group } \\
n=83\end{array}$ & $P$ value \\
\hline \multicolumn{4}{|c|}{ In-ICU mortality } \\
\hline General & $* 17(25)$ & *7 (11) & 0.04 \\
\hline Circulatory & $7 / 23(30)$ & $1 / 17(6)$ & 0.11 \\
\hline Respiratory & $10 / 44(23)$ & $6 / 45(13)$ & 0.25 \\
\hline \multicolumn{4}{|c|}{ In-hospital mortality } \\
\hline General & $29(35)$ & $14(17)$ & 0.007 \\
\hline Circulatory & $12 / 26(46)$ & $3 / 22(14)$ & 0.02 \\
\hline Respiratory & $17 / 56(30)$ & 11/61 (18) & 0.12 \\
\hline
\end{tabular}

* Patients admitted in ICU: control group $n=67$ and POCUS group $n=62$

${ }^{a}$ Data are expressed as no. (\%) of participants

certainly not on the ward. One multicenter, prospective, controlled study conducted in hypotensive patients in the emergency department showed no association between the use of POCUS and patient outcomes [34]. However, the treatment provided was similar in the two arms, suggesting that benefit from the use of POCUS was not maximalized. At the same time, the study was stopped prematurely because the treating physicians felt it was unethical to resuscitate patients without the use of POCUS. Conversely, two studies suggested an association between the use of ultrasound and patient outcomes. In a before-after study, Kanji et al. found that limited echocardiography-guided therapy was associated with a reduction in mortality in patients with shock [35]. Patients who were treated based on echocardiography findings received less intravenous fluids. In a retrospective analysis of a multicenter database, Feng et al. also showed that the use of echocardiography in septic patients was associated with reduced mortality. However, treatments based on echocardiography findings resulted in more fluids in the first $24 \mathrm{~h}$ [36]. Regardless of the amount of fluids transfused, the findings in these studies are similar to ours with regards to the association with improved outcomes.

Ray et al. showed that inappropriate diagnosis and treatment of patients in acute respiratory failure are associated with increased mortality [37]. We observed earlier intervention in the POCUS group, as did others [7]. The lower mortality rates observed with the use of POCUS may stem from earlier intervention. POCUS use reduces diagnostic uncertainty [26] and can identify life-threatening conditions that would otherwise be missed [38]. The use of a handheld POCUS device has also been shown to be particularly efficient for cardiac diagnosis [39-42]. In the current study, the reduced use of invasive mechanical ventilation, steroids and vasopressors in the POCUS group suggests more targeted treatment in patients with an underlying cardiac cause for deterioration. In our study, the proportion of appropriate intervention at the bedside was similar in the two groups but the number of interventions was significantly higher in the control group than in the POCUS group. Thus, POCUS makes it possible to adjust early the best treatment.

Our study has several limitations. It is a single-center study, which limits the extrapolation of our findings. However, the in-hospital mortality of our control group (35\%) is similar to that described in other studies and aligns with the predicted mortality of our cohort [43, 44]. Our study was only controlled. Although the two groups were well balanced, the patients in the control group may have been sicker (as evidenced by their lower MAP, higher mottling scores and higher oxygen flows), and regarding the outcomes, our results were not significant after matching. However, we adjusted the mortality rates to account for this potential bias and our results remained significant in most of the models chosen. There was no crossover between the two RRT. The probability of a specific team effect is reduced by the fact that during the study period a large number of seniors and juniors randomly composed each RRT. We focused our study on the first treatment administered by the RRT and not on treatment during the first $24 \mathrm{~h}$, which may have 
differed in the two groups. However, our patients were managed in the same institution, by similar ward physicians, surgeons and intensivists. Finally, the contribution of POCUS cannot be separated from that of the actual treatment protocol in our study. Regardless, the use of a combined strategy seems to be associated with improved performance.

\section{Conclusion}

Our clinical trial suggests that protocolized use of a handheld POCUS device at the bedside in the ward by RRT may improve the proportion of adequate diagnosis, the time to initial treatment and perhaps also survival of ward patients developing acute respiratory and/or circulatory failure. However, because the control group was slightly sicker than the POCUS group, our results need to be confirmed by future multicenter, randomized, controlled trials.

\section{Supplementary information}

The online version contains supplementary material available at https://doi. org/10.1186/s13054-021-03466-z.

Additional file 1. Additional Table 1: Glossary of terms used in the study (supplement material).

Additional file 2. Additional Table 2: List of evocated diagnosis (A) and care management (B) after management at the bedside in ward (supplement material).

Additional file 3. Additional Table 3: Summary of patients' characteristics (supplement material).

Additional file 4. Additional Method: Details of propensity score (supplement material).

Additional file 5. Additional Table 4: Summary of senior and resident ICU physicians medical experience between the two groups (supplement material).

Additional file 6. Additional Table 5: Propensity score between the two groups (supplement material).

Additional file 7. Additional Table 6: POCUS effect group on in-hospital mortality (logistic regressions, sensitivity analyses) (supplement material).

\section{Abbreviations}

Cl: Confidence interval; HHUD: Handheld ultrasound devices; ICU: Intensive care unit; IQR: Interquartile range; MAP: Mean arterial pressure; OR: Odds ratio; POCUS: Point-of-care ultrasound; RRT: Rapid response team; SAPS: Simplified Acute Physiology Score.

\section{Acknowledgements}

None.

\section{Authors' contributions}

LZ and $A L$ are joint first authors and contributed equally. LZ, AL and ML were responsible for study design, data acquisition, analysis and interpretation and for preparing the first draft of the manuscript. SH, BP, MDB and AB were responsible for conducting the study. $G D$ and $B C$ were responsible for recruiting study participants and clinical input in the study design. KB was responsible for statistical analysis. XB, SE, LP and ML helped write the manuscript. All authors had an opportunity to review the manuscript and approved its final submitted version.
Funding

None.

Availability of data and materials

All the data generated and analyzed during this study are included in this published article.

\section{Ethics approval and consent to participate}

The study was approved by the Committee for Research Ethics of French Society of Anesthesia \& Intensive Care Medicine (CERAR no. IRB 00010254-2016-

122). The study was also registered in ClinicalTrials.gov (NCT02967809).

\section{Consent for publication}

This manuscript has been read and its submission approved by all the coauthors. The patients were prospectively included after providing informed consent.

\section{Competing interests}

LZ and XB received fees from General Electric Healthcare for ultrasound teaching.

\section{Author details}

${ }^{1}$ Assistance Publique Hôpitaux de Marseille, Department of Anesthesiology and Intensive Care, Hôpital Nord, Aix Marseille University, 13015 Marseille, France. ${ }^{2}$ Assistance Publique Hôpitaux de Marseille, Service de Médecine Intensive - Réanimation, Hôpital Nord, Aix Marseille University, 13015 Marseille, France. ${ }^{3}$ Centre D'Etudes et de Recherches sur les Services de Santé et Qualité, Faculté de Médecine, Aix-Marseille Université, 13005 Marseille, France. ${ }^{4}$ Assistance Publique Hôpitaux de Marseille, Service d'Imagerie Médicale, Hôpital Nord, Aix Marseille University, 13015 Marseille, France. ${ }^{5}$ Assistance Publique Hôpitaux de Marseille, Service des Explorations Fonctionnelles Respiratoires, Aix Marseille University, 13015 Marseille, France. ${ }^{6}$ Center for Cardiovascular and Nutrition Research (C2VN), INSERM, INRA, Aix Marseille Université, 13005 Marseille, France. ${ }^{7}$ Intensive Care Unit, Department of Anesthesiology, Emergency and Critical Care Medicine, University Hospital Nimes, 30000 Nîmes, France. ${ }^{8}$ Surgical Intensive Care Unit, Shaare Zedek Medical Center, Faculty of Medicine, Hebrew University, Jerusalem, Israel.

Received: 25 September 2020 Accepted: 12 January 2021

Published online: 22 January 2021

\section{References}

1. Jones DA, DeVita MA, Bellomo R. Rapid-response teams. N Engl J Med. 2011;365(2):139-46.

2. Brennan TA, Localio AR, Leape LL, Laird NM, Peterson L, Hiatt HH, et al. Identification of adverse events occurring during hospitalization. A crosssectional study of litigation, quality assurance, and medical records at two teaching hospitals. Ann Intern Med. 1990;112(3):221-6.

3. Devita MA, Bellomo R, Hillman K, Kellum J, Rotondi A, Teres D, et al. Findings of the first consensus conference on medical emergency teams. Crit Care Med. 2006;34(9):2463-78.

4. Maharaj R, Raffaele I, Wendon J. Rapid response systems: a systematic review and meta-analysis. Crit Care. 2015;19:254.

5. Al Deeb M, Barbic S, Featherstone R, Dankoff J, Barbic D. Point-of-care ultrasonography for the diagnosis of acute cardiogenic pulmonary edema in patients presenting with acute dyspnea: a systematic review and meta-analysis. Acad Emerg Med. 2014;21 (8):843-52.

6. Joseph MX, Disney PJS, Da Costa R, Hutchison SJ. Transthoracic echocardiography to identify or exclude cardiac cause of shock. Chest. 2004;126(5):1592-7.

7. Laursen CB, Sloth E, Lassen AT, dePont CR, Lambrechtsen J, Madsen PH, et al. Point-of-care ultrasonography in patients admitted with respiratory symptoms: a single-blind, randomised controlled trial. Lancet Respir Med. 2014;2(8):638-46.

8. Zanobetti M, Scorpiniti M, Gigli C, Nazerian P, Vanni S, Innocenti F, et al. Point-of-care ultrasonography for evaluation of acute dyspnea in the ED. Chest. 2017;151(6):1295-301. 
9. Bataille B, Riu B, Ferre F, Moussot PE, Mari A, Brunel E, et al. Integrated use of bedside lung ultrasound and echocardiography in acute respiratory failure: a prospective observational study in ICU. Chest 2014;146(6):1586-93.

10. Biais M, Carrié C, Delaunay F, Morel N, Revel P, Janvier G. Evaluation of a new pocket echoscopic device for focused cardiac ultrasonography in an emergency setting. Crit Care. 2012;16(3):R82.

11. Dalen H, Gundersen GH, Skjetne K, Haug HH, Kleinau JO, Norekval TM, et al. Feasibility and reliability of pocket-size ultrasound examinations of the pleural cavities and vena cava inferior performed by nurses in an outpatient heart failure clinic. Eur J Cardiovasc Nurs. 2015;14(4):286-93.

12. Sforza A, Mancusi C, Carlino MV, Buonauro A, Barozzi M, Romano G, et al. Diagnostic performance of multi-organ ultrasound with pocket-sized device in the management of acute dyspnea. Cardiovasc Ultrasound. 2017;15(1):16.

13. Busch M. Portable ultrasound in pre-hospital emergencies: a feasibility study. Acta Anaesthesiol Scand. 2006;50(6):754-8.

14. Toulouse E, Masseguin C, Lafont B, McGurk G, Harbonn A, Roberts A, et al. French legal approach to clinical research. Anaesth Crit Care Pain Med. 2018;37(6):607-14.

15. von Elm E, Altman DG, Egger M, Pocock SJ, Gøtzsche PC, Vandenbroucke $J \mathrm{~J}$, et al. The strengthening the reporting of observational studies in epidemiology (STROBE) statement: guidelines for reporting observational studies. J Clin Epidemiol. 2008;61 (4):344-9.

16. Expert Round Table on Echocardiography in ICU. International consensus statement on training standards for advanced critical care echocardiography. Intensive Care Med. 2014;40(5):654-66.

17. Zieleskiewicz L, Bouvet L, Einav S, Duclos G, Leone M. Diagnostic pointof-care ultrasound: applications in obstetric anaesthetic management. Anaesthesia. 2018;73(10):1265-79.

18. Lichtenstein DA, Mezière GA. Relevance of lung ultrasound in the diagnosis of acute respiratory failure: the BLUE protocol. Chest. 2008;134(1):117-25.

19. Wu TS. The CORE scan: concentrated overview of resuscitative efforts. Crit Care Clin. 2014;30(1):151-75.

20. Liteplo A, Noble V, Atkinson P. My patient has no blood pressure: pointof-care ultrasound in the hypotensive patient - FAST and RELIABLE. Ultrasound. 2012;20(1):64-8.

21. Manno E, Navarra M, Faccio L, Motevallian M, Bertolaccini L, Mfochivè A, et al. Deep impact of ultrasound in the intensive care unit: the 'ICU-sound' protocol. Anesthesiology. 2012;117(4):801-9.

22. D'Agostino RB. Propensity score methods for bias reduction in the comparison of a treatment to a non-randomized control group. Stat Med. 1998;17(19):2265-81.

23. Silva S, Biendel C, Ruiz J, Olivier M, Bataille B, Geeraerts T, et al. Usefulness of cardiothoracic chest ultrasound in the management of acute respiratory failure in critical care practice. Chest. 2013;144(3):859-65.

24. Pivetta E, Goffi A, Lupia E, Tizzani M, Porrino G, Ferreri E, et al. Lung ultrasound-implemented diagnosis of acute decompensated heart failure in the ED: a SIMEU multicenter study. Chest. 2015;148(1):202-10.

25. Volpicelli G, Lamorte A, Tullio M, Cardinale L, Giraudo M, Stefanone V, et al. Point-of-care multiorgan ultrasonography for the evaluation of undifferentiated hypotension in the emergency department. Intensive Care Med. 2013;39(7):1290-8.

26. Shokoohi H, Boniface KS, Pourmand A, Liu YT, Davison DL, Hawkins KD, et al. Bedside ultrasound reduces diagnostic uncertainty and guides resuscitation in patients with undifferentiated hypotension. Crit Care Med. 2015;43(12):2562-9.

27. Shokoohi H, Boniface KS, Zaragoza M, Pourmand A, Earls JP. Point-of-care ultrasound leads to diagnostic shifts in patients with undifferentiated hypotension. Am J Emerg Med. 2017;35(12):1984.e3-1984.e7.

28. Zieleskiewicz L, Muller L, Lakhal K, Meresse Z, Arbelot C, Bertrand P-M et al. Point-of-care ultrasound in intensive care units: assessment of 1073 procedures in a multicentric, prospective, observational study. Intensive Care Med. 2015;41(9):1638-47.
29. Narula J, Chandrashekhar Y, Braunwald E. Time to add a fifth pillar to bedside physical examination: inspection, palpation, percussion, auscultation, and insonation. JAMA Cardiol. 2018;3(4):346-50.

30. Mjølstad OC, Andersen GN, Dalen H, Graven T, Skjetne K, Kleinau JO, et al. Feasibility and reliability of point-of-care pocket-size echocardiography performed by medical residents. Eur Heart J Cardiovasc Imaging. 2013;14(12):1195-202.

31. Pujol S, Laurent J, Markarian T, Claret P-G, Lefrant JY, Roger C, et al. Compression with a pocket-sized ultrasound device to diagnose proximal deep vein thrombosis. Am J Emerg Med. 2018;36(7):1262-4.

32. Cardim N, Dalen H, Voigt J-U, Ionescu A, Price S, Neskovic AN, et al. The use of handheld ultrasound devices: a position statement of the European Association of Cardiovascular Imaging (2018 update). Eur Heart J Cardiovasc Imaging. 2019;20(3):245-52.

33. Bobbia X, Pradeilles C, Claret PG, Soullier C, Wagner P, Bodin Y, et al. Does physician experience influence the interpretability of focused echocardiography images performed by a pocket device? Scand J Trauma Resusc Emerg Med. 2015;23:52.

34. Atkinson PR, Milne J, Diegelmann L, Lamprecht H, Stander M, Lussier D, et al. Does point-of-care ultrasonography improve clinical outcomes in emergency department patients with undifferentiated hypotension? An international randomized controlled trial from the SHoC-ED investigators. Ann Emerg Med. 2018;72(4):478-89.

35. Kanji HD, McCallum J, Sirounis D, MacRedmond R, Moss R, Boyd JH. Limited echocardiography-guided therapy in subacute shock is associated with change in management and improved outcomes. J Crit Care. 2014:29(5):700-5.

36. Feng M, McSparron Jl, Kien DT, Stone DJ, Roberts DH, Schwartzstein RM, et al. Transthoracic echocardiography and mortality in sepsis: analysis of the MIMIC-III database. Intensive Care Med. 2018;44(6):884-92.

37. Ray P, Birolleau S, Lefort Y, Becquemin M-H, Beigelman C, Isnard R, et al. Acute respiratory failure in the elderly: etiology, emergency diagnosis and prognosis. Crit Care. 2006;10(3):R82.

38. Laursen CB, Sloth E, Lambrechtsen J, Lassen AT, Madsen PH, Henriksen DP, et al. Focused sonography of the heart, lungs, and deep veins identifies missed life-threatening conditions in admitted patients with acute respiratory symptoms. Chest. 2013;144(6):1868-75.

39. Panoulas VF, Daigeler A-L, Malaweera ASN, Lota AS, Baskaran D, Rahman $S$, et al. Pocket-size hand-held cardiac ultrasound as an adjunct to clinical examination in the hands of medical students and junior doctors. Eur Heart J Cardiovasc Imaging. 2013;14(4):323-30.

40. Decara JM, Kirkpatrick JN, Spencer KT, Ward RP, Kasza K, Furlong K, et al. Use of hand-carried ultrasound devices to augment the accuracy of medical student bedside cardiac diagnoses. J Am Soc Echocardiogr. 2005;18(3):257-63.

41. Kobal SL, Trento L, Baharami S, Tolstrup K, Naqvi TZ, Cercek B, et al. Comparison of effectiveness of hand-carried ultrasound to bedside cardiovascular physical examination. Am J Cardiol. 2005;96(7):1002-6.

42. Stokke TM, Ruddox V, Sarvari SI, Otterstad JE, Aune E, Edvardsen T. Brief group training of medical students in focused cardiac ultrasound may improve diagnostic accuracy of physical examination. J Am Soc Echocardiogr. 2014;27(11):1238-46.

43. Tirkkonen J, Tamminen T, Skrifvars MB. Outcome of adult patients attended by rapid response teams: a systematic review of the literature. Resuscitation. 2017:112:43-52.

44. Le Gall JR, Lemeshow S, Saulnier F. A new simplified acute physiology score (SAPS II) based on a European/North American multicenter study. JAMA. 1993;270(24):2957-63.

\section{Publisher's Note}

Springer Nature remains neutral with regard to jurisdictional claims in published maps and institutional affiliations. 